\title{
The transcription factor BACH2 promotes tumor immunosuppression
}

\author{
Rahul Roychoudhuri, ${ }^{1,2}$ Robert L. Eil, ${ }^{1}$ David Clever, ${ }^{1}$ Christopher A. Klebanoff, ${ }^{1}$ Madhusudhanan Sukumar, ${ }^{1}$ \\ Francis M. Grant, ${ }^{2}$ Zhiya Yu, ${ }^{1}$ Gautam Mehta, ${ }^{1}$ Hui Liu, ${ }^{3}$ Ping Jin, ${ }^{3}$ Yun Ji, ${ }^{1}$ Douglas C. Palmer, ${ }^{1}$ Jenny H. Pan, ${ }^{1}$ \\ Anna Chichura, ${ }^{1}$ Joseph G. Crompton, ${ }^{1}$ Shashank J. Patel, ${ }^{1}$ David Stroncek, ${ }^{3}$ Ena Wang, ${ }^{4}$ Francesco M. Marincola, ${ }^{4}$ \\ Klaus Okkenhaug, ${ }^{2}$ Luca Gattinoni, ${ }^{1}$ and Nicholas P. Restifo ${ }^{1}$ \\ 1National Cancer Institute, NIH, Bethesda, Maryland, USA. Babraham Institute, Babraham Research Campus, Cambridge, United Kingdom. 'epartment of Transfusion Medicine, Clinical Center, \\ NIH, Bethesda, Maryland, USA. ${ }^{4}$ Research Branch, Sidra Medical and Research Centre, Doha, Qatar.
}

The immune system has a powerful ability to recognize and kill cancer cells, but its function is often suppressed within tumors, preventing clearance of disease. Functionally diverse innate and adaptive cellular lineages either drive or constrain immune reactions within tumors. The transcription factor (TF) BACH2 regulates the differentiation of multiple innate and adaptive cellular lineages, but its role in controlling tumor immunity has not been elucidated. Here, we demonstrate that BACH2 is required to establish immunosuppression within tumors. Tumor growth was markedly impaired in Bach2-deficient mice and coincided with intratumoral activation of both innate and adaptive immunity. However, augmented tumor clearance in the absence of Bach2 was dependent upon the adaptive immune system. Analysis of tumor-infiltrating lymphocytes from Bach2-deficient mice revealed high frequencies of rapidly proliferating effector CD4+ and CD8 ${ }^{+} \mathrm{T}$ cells that expressed the inflammatory cytokine IFN- $\gamma$. Effector T cell activation coincided with a reduction in the frequency of intratumoral Foxp ${ }^{+}$ Tregs. Mechanistically, BACH2 promoted tumor immunosuppression through Treg-mediated inhibition of intratumoral $\mathrm{CD}^{+} \mathrm{T}$ cells and IFN- $\gamma$. These findings demonstrate that $\mathrm{BACH} 2$ is a key component of the molecular program of tumor immunosuppression and identify therapeutic targets for the reversal of immunosuppression in cancer.

\section{Introduction}

While the immune system has a powerful ability to recognize and kill cancer cells, its function is often suppressed, preventing clearance of disease. A variety of innate and adaptive immune lineages causes immunosuppression within tumors, including immature DCs, plasmacytoid DCs, myeloid-derived suppressor cells, CD $4^{+}$ Foxp3 ${ }^{+}$Tregs, IL-10-secreting type-I Tregs, and CD1d-restricted natural killer T cells (1-5). Thus, a relatively well-characterized network of innate and adaptive immunosuppressive cell types drives immunosuppression, but molecular mechanisms required for the development of immunosuppressive responses within tumors are poorly elucidated. TFs play key roles in cellular differentiation and bind to regulatory DNA to control gene expression. BACH2 is a 92-kDa transcription factor (TF) of the basic leucine zipper family and functions within multiple innate and adaptive lineages to control immune function. In B cells, $\mathrm{BACH} 2$ is critical for somatic hypermutation and class-switch recombination, and its absence leads to impaired formation of class-switched antibody responses $(6,7)$. In $\mathrm{CD}^{+}{ }^{+} \mathrm{T}$ cells, BACH 2 promotes the development of Foxp $3^{+}$ Tregs by suppressing effector cell transcriptional programs $(8,9)$. $\mathrm{BACH} 2$ is also required for normal development and function of alveolar macrophages (10). Consistent with its role in regulating

Authorship note: R. Roychoudhuri, R.L. Eil, and D. Clever contributed equally to this work. Conflict of interest: The authors have declared that no conflict of interest exists. Submitted: May 19, 2015; Accepted: November 16, 2015

Reference information: / Clin Invest. 2016;126(2):599-604. doi:10.1172/JCI82884. the development and function of diverse immune cell types, the $B A C H 2$ gene in humans is a prominent susceptibility locus for multiple autoimmune and allergic diseases (11-14). Thus, BACH2 functions in a variety of cellular lineages that can either promote or suppress immune responses against tumors. However, its function in controlling tumor immunity has not been elucidated.

In this study, we have found that $\mathrm{BACH} 2$ is required to establish immunosuppression within tumors. We found that growth of B16 melanoma and EL-4 lymphoma tumors was markedly impaired in Bach2-deficient mice and coincided with intratumoral activation of both innate and adaptive immunity. However, augmented tumor clearance in the absence of Bach2 was dependent upon adaptive immunity. Analysis of tumor-infiltrating lymphocytes in Bach2-deficient mice revealed high frequencies of rapidly proliferating $\mathrm{CD}^{+}$and $\mathrm{CD}^{+}$effector cells expressing the inflammatory cytokine IFN- $\gamma$. Lymphocyte activation coincided with reduction in the frequency of intratumoral CD $4^{+}$Foxp $3^{+}$Tregs. Treg-dependent inhibition of intratumoral $\mathrm{CD}^{+} \mathrm{T}$ cells and IFN- $\gamma$ was required for $\mathrm{BACH} 2-$ mediated tumor immunosuppression. These findings identify BACH2 as a key component of the molecular program of tumor immunosuppression and identify a target for therapies aimed at reversing immunosuppression in cancer.

\section{Results and Discussion}

BACH2 promotes tumor immunosuppression. To determine the function of $\mathrm{BACH} 2$ in regulating tumor immunity, we implanted syngeneic B16 melanoma cells subcutaneously into littermate 

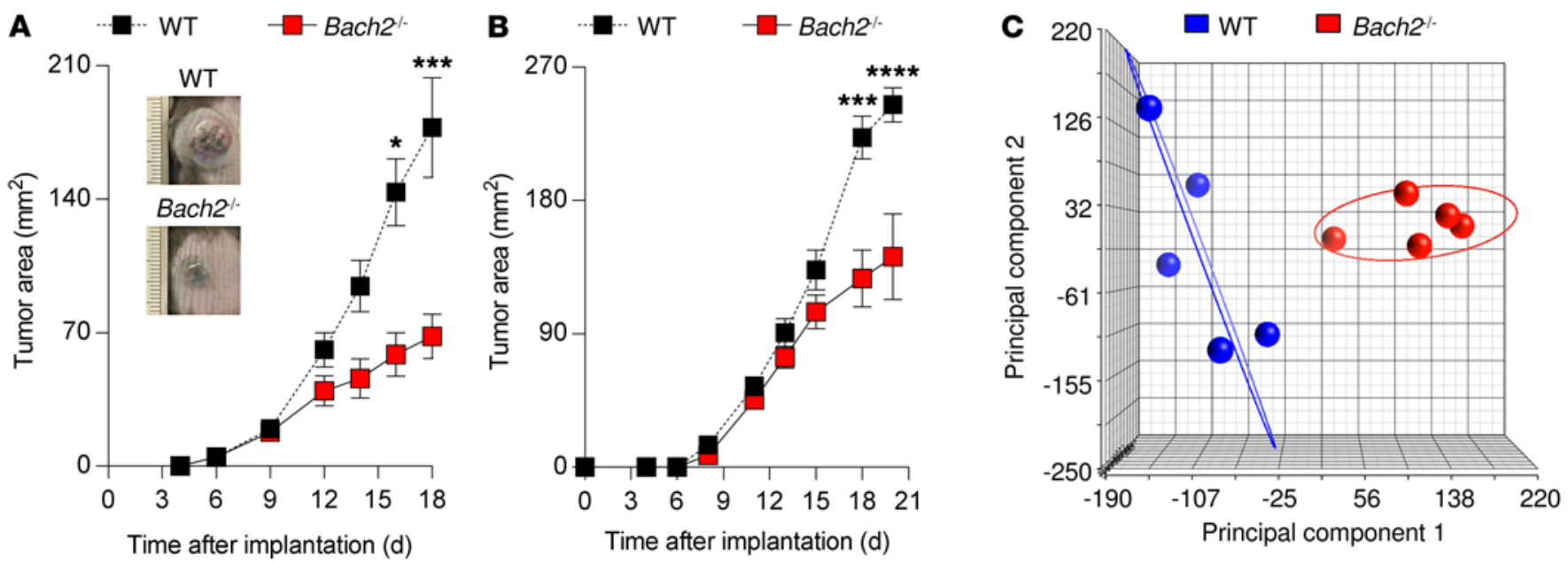

D

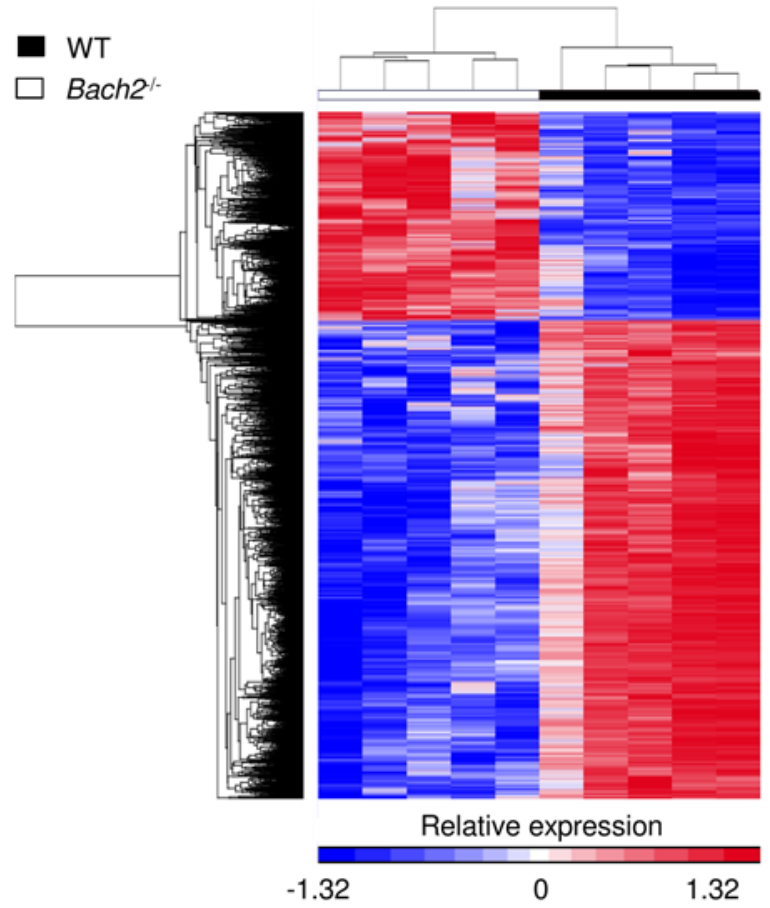

$\mathbf{E}$

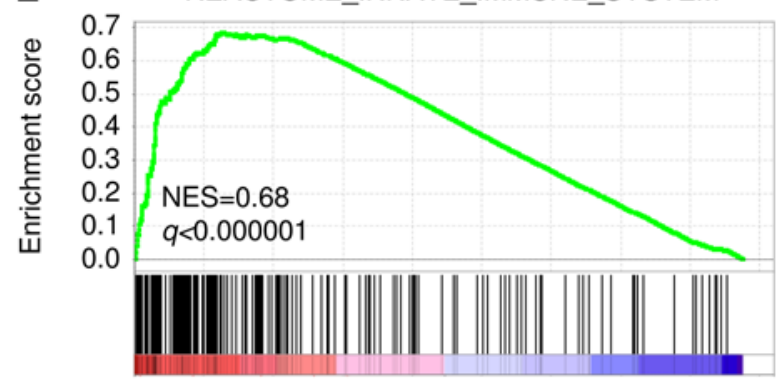

$\mathbf{F}$

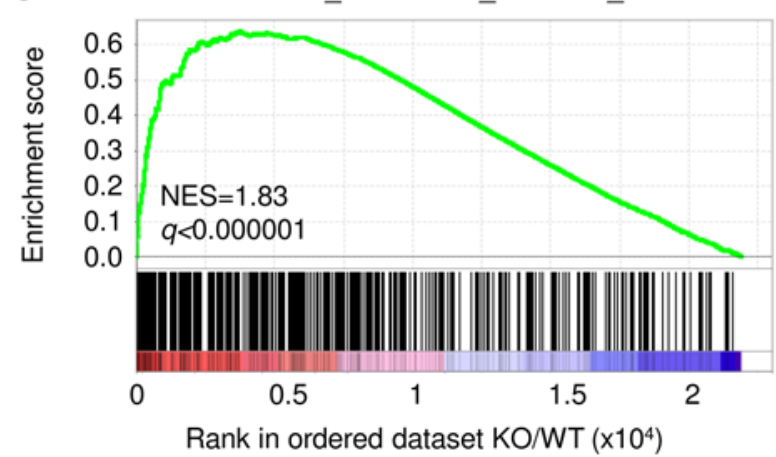

Figure 1. BACH2 promotes immunosuppression within tumors. (A and B) Growth of subcutaneous (A) B16 and (B) EL-4 tumors in littermate Bach2 ${ }^{+/+}$(WT) and Bach2 $2^{-/-}$mice at indicated time points following implantation. (A) Representative photographs of tumors at day 18 after implantation (inset). Rulers show millimeters. Error bars represent mean \pm SEM. ${ }^{*} P<0.05 ;{ }^{* * *} P<0.005 ;{ }^{* * *} P<0.001$, 2-tailed Student's $t$ tests. (C) Principal component analysis of transcriptional profiles from tumors of $\mathrm{Bach}^{+/+}$(blue) and Bach2 ${ }^{-/-}$(red) mice at day 18 following implantation of B16 tumor cells. Principal component 3 is indicated by the axis perpendicular to the $x$ and $y$ axes. (D) Global differences in transcriptional profiles of tumors from Bach2-deficient and WT mice. 3,623 differentially expressed genes identified in the analysis (adjusted $P<0.05$; fold change $>2$, 2-tailed Student's $t$ tests) are shown. (E and $\mathbf{F}$ ) Gene set enrichment analysis of global transcriptional differences between tumors from Bach2-deficient and WT mice. Positions of genes from indicated gene sets within a list of gene expression differences rank ordered by fold change are indicated. Kolmogorov-Smirnov statistic was used to calculate statistical significance. All data are representative of $\geq 2$ independent experiments. NES, normalized enrichment score.

WT or Bach2-deficient mice and measured tumor size at serial time points following implantation. Strikingly, we noted reduced growth of B16 tumors in Bach2-deficient animals (Figure 1A). Similar observations were made using the EL-4 lymphoma model (Figure 1B). To determine the basis for impaired tumor growth in Bach2-deficient animals, we performed whole-tumor transcriptional profiling of B16 tumors from WT and Bach2-deficient animals. Whole-transcriptome profiles of tumors from Bach2-defi- cient animals were uniformly distinct from those of WT animals (Figure 1C). Analysis of transcriptional profiles for global differences in gene expression identified 3,623 differentially expressed transcripts (Supplemental Table 1; supplemental material available online with this article; doi:10.1172/JCI82884DS1), which enabled tumors to be distinguished based upon hierarchical cluster analysis (Figure 1D). Gene set enrichment analysis (15) indicated induction of transcriptional signatures of both innate and 
A

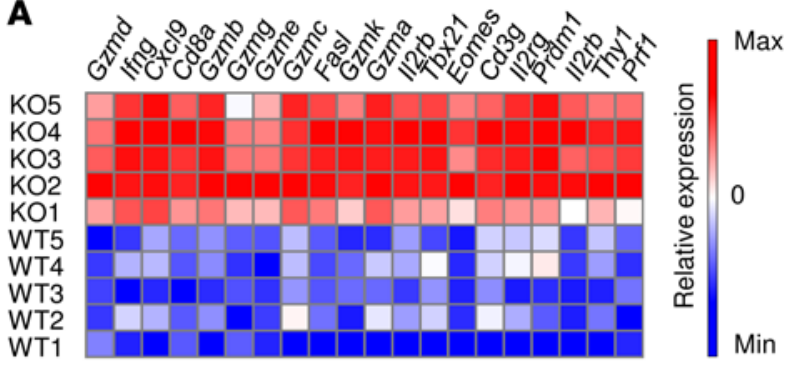

B

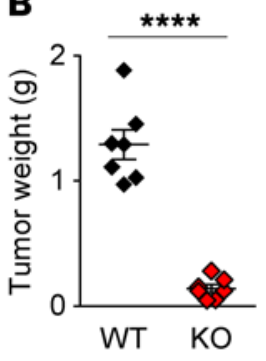

C

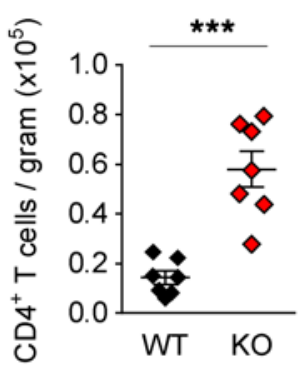

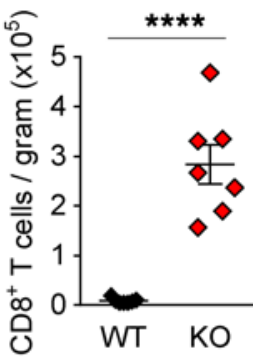

D

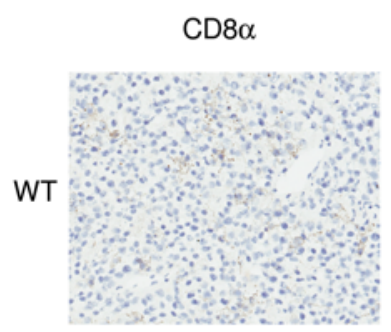

$\mathrm{KO}$

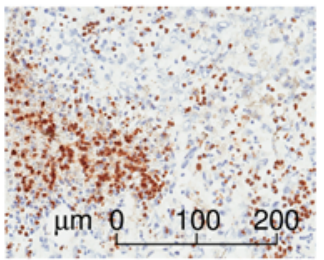

E
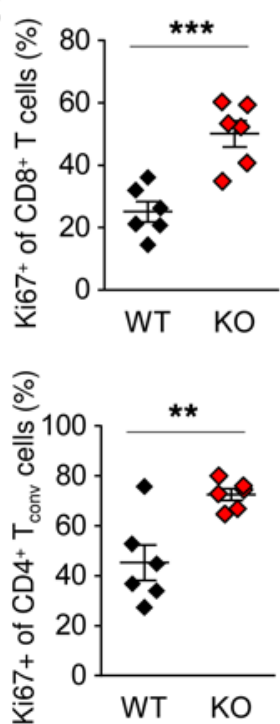

F

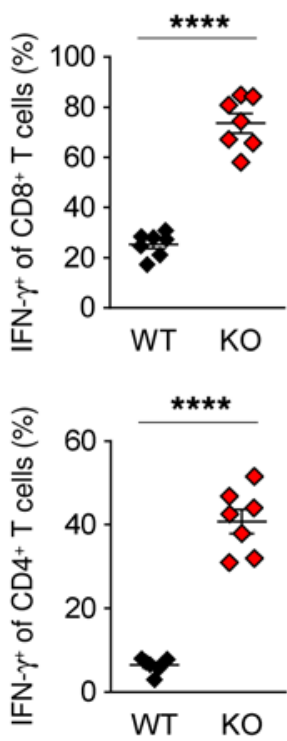

G

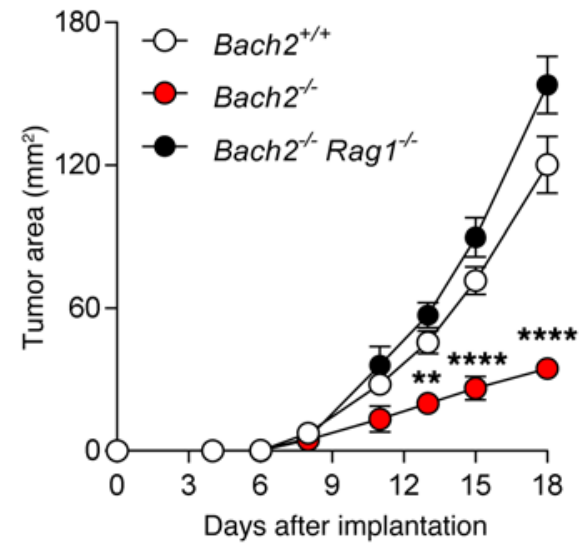

Figure 2. BACH2 constrains T cell-mediated antitumor immunity. (A) Heatmap indicating relative expression of indicated genes within global tran-

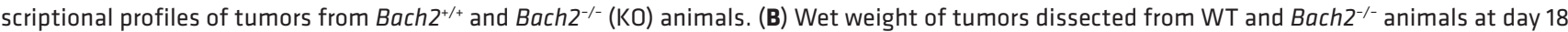
following implantation. (C) Density of CD4+ and CD8+ $T$ cells normalized by tumor mass. (D) Immunohistochemical analysis of CD8 ${ }^{+} \mathrm{T}$ cells in sections of B16 tumors from mice of indicated genotypes at day 18 after implantation. Scale bar: $200 \mu \mathrm{m}$. (E) Frequency of Ki67 $7^{+}$cells among CD8 $8^{+}$T cells and CD4+ Foxp3- conventional T (Tconv) cells at day 18 after implantation. (F) IFN- $\gamma$ expression among intratumoral CD4+ and CD8 ${ }^{+}$T cells at day 18 after implantation. (G) Growth of subcutaneous B16 tumors in littermate mice of the indicated genotypes at selected time points following implantation. Significant differences from growth in $\mathrm{Bach}^{+/+}$mice are indicated. Error bars represent mean $\pm \mathrm{SEM}$. Results are representative of $\geq 2$ independent experiments. ${ }^{* *} P<0.01 ;{ }^{* *} P<0.005 ;{ }^{* * *} P<0.001,2$-tailed Student's $t$ tests.

adaptive immune activation in tumors from Bach2-deficient mice (Figure 1, E and F, and Supplemental Tables 2-4). Collectively, these data indicate that $\mathrm{BACH} 2$ promotes tumor growth in a tumor cell-extrinsic fashion and suppresses induction of transcriptional profiles indicative of innate and adaptive immune.

BACH2 suppresses adaptive immunity to promote immunosuppression within tumors. $\mathrm{CD}^{+}$and $\mathrm{CD} 8^{+} \mathrm{T}$ cells form a key component of adaptive immune responses against tumors. Whole-tumor transcriptional profiles indicated striking induction of genes associated with $\mathrm{CD}^{+}$and $\mathrm{CD} 8^{+}$effector $\mathrm{T}$ cells in tumors from Bach2-deficient mice. In particular, we noted elevated expression of genes that are expressed by T cells (including Cd3g, Il2rg, Thy1, and $C d 8 a)(16)$ or associated with effector differentiation (including Tbx21 and Prdm1) $(17,18)$ and function (including Ifng, Gzmb, and Fasl) (ref. 16 and Figure 2A). We therefore measured infiltration of $\mathrm{CD}^{+}$and $\mathrm{CD}^{+} \mathrm{T}$ cells within tumors using flow cytometry. Normalized for differences in tumor mass (Figure 2B), the density of both $\mathrm{CD} 4^{+}$and $\mathrm{CD}^{+} \mathrm{T}$ cells was higher in tumors from Bach2-deficient animals (Figure 2C). Increased density of $\mathrm{CD}^{+}$ and $\mathrm{CD}^{+} \mathrm{T}$ cells was also apparent from immunohistochemical analysis of tumor sections (Figure 2D and Supplemental Figure 1A). Consistent with their increased density, we observed elevated frequencies of proliferating $\mathrm{Ki}^{+} 7^{+}$cells among both $\mathrm{CD} 8^{+}$and $\mathrm{CD}^{+}{ }^{+}$Foxp3 ${ }^{-}$effector T cells within tumors from Bach2-deficient mice (Figure 2E). Moreover, intracellular cytokine staining following brief restimulation of tumor-infiltrating cells ex vivo showed strikingly elevated frequencies of $\mathrm{CD}^{+}$and $\mathrm{CD}^{+} \mathrm{T}$ cells expressing the effector cytokine IFN- $\gamma$ within tumors from Bach2-deficient mice (Figure 2F and Supplemental Figure 1B). Thus, BACH2 limits the proliferation of $\mathrm{CD} 8^{+}$and $\mathrm{CD} 4^{+}$effector $\mathrm{T}$ cells and their expression of effector cytokines within tumors.

The protein encoded by recombination-activating gene 1 (Rag1) is required for rearrangement of $\mathrm{T}$ and $\mathrm{B}$ cell receptor loci, and its loss results in a complete defect in generation of mature $\mathrm{T}$ and B lymphocytes (19). To test whether decreased tumor growth in the absence of BACH2 was dependent upon lymphocytes, we measured growth kinetics of subcutaneously implanted B16 melanoma cells in Bach2 Rag1 double-deficient animals (Figure 2G). 
A

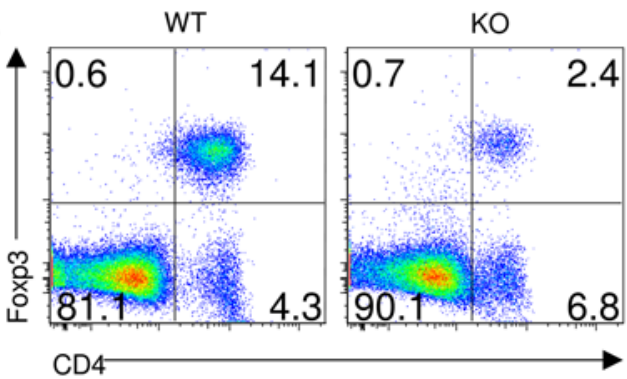

B

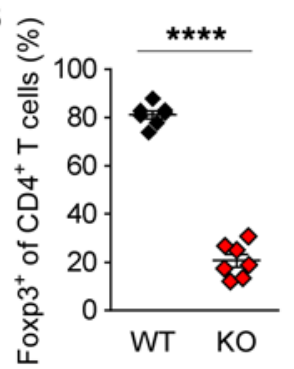

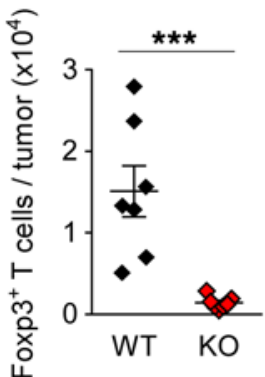

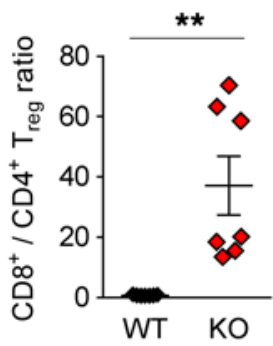

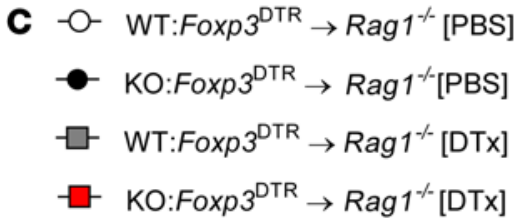

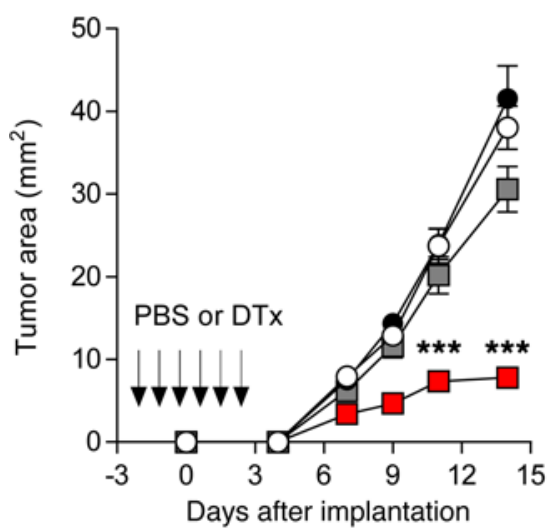

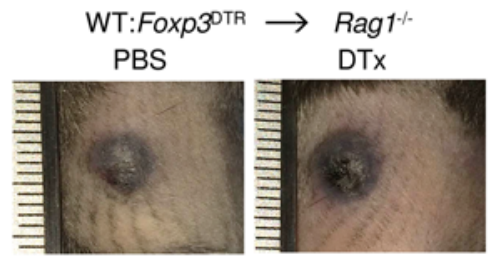

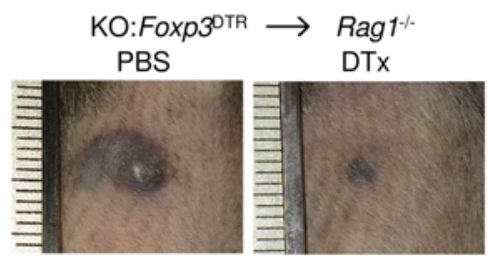

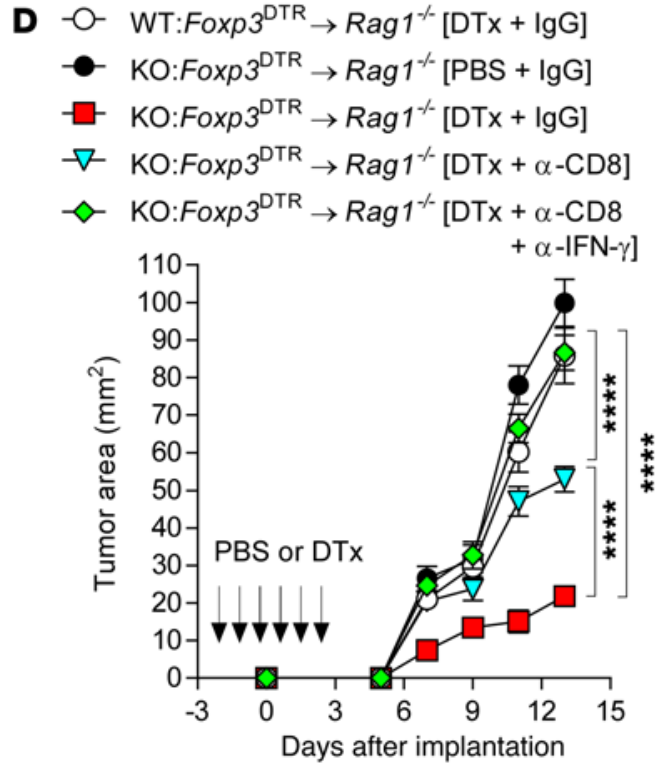

Figure 3. BACH2 promotes tumor growth through Treg-mediated suppression of intratumoral CD8 ${ }^{+} \mathbf{T}$ cells and IFN- $\gamma$. (A) Representative flow cytometry and (B) replicate measurements of CD4 and Foxp3 expression among T cells infiltrating B16 tumors at day 18 following implantation. Numbers in gates represent percentages of gated cells. (C) Rag1/-- mice were reconstituted with 1:1 mixtures of BM cells from Foxp $3^{\text {DTR }}$ mice and either WT or Bach2-deficient (KO) mice and administered PBS or DTx at indicated time points relative to implantation. Tumor growth was measured after subcutaneous implantation of $1.25 \times$ $10^{5}$ B16 cells at indicated time points following implantation. Significant differences in tumor area compared with the WT:Foxp $3^{\text {DTR }} \rightarrow$ Rag ${ }^{-1-}$ (PBS) group are indicated. Representative photographs of tumors at day 18 after implantation are shown. Rulers show millimeters. (D) Rag $1^{-1-}$ mice were reconstituted with 1:1 mixtures of BM cells from Bach2-deficient and Foxp $3^{\text {DTR }}$ mice and administered PBS or DTx in conjunction with $\alpha$-CD8- and/or $\alpha$-IFN- $\gamma$-depleting monoclonal antibodies or isotype control antibodies (IgG) at indicated time points relative to implantation. Tumor growth was measured at indicated time points after subcutaneous implantation of $2.5 \times 10^{5}$ B16 cells. All data are representative of $\geq 2$ independent experiments. Error bars represent mean $\pm \mathrm{SEM}$. ${ }^{* *} P<0.01$; ${ }^{* * *} P<0.005 ;{ }^{* * *} P<0.001,2$-tailed Student's $t$ tests.

Consistent with our previous observations, tumor growth was markedly impaired in Bach2-deficient animals. However, this was dependent upon the activity of lymphocytes, since augmented tumor clearance was not seen in Bach2 Rag1 double-deficient animals. Thus, decreased tumor growth in the absence of $\mathrm{BACH} 2$ requires the adaptive immune system.

BACH2 causes tumor growth through Treg-mediated suppression of $C D 8^{+} T$ cells. While $\mathrm{CD} 8^{+} \mathrm{T}$ cells primarily function to promote tumor clearance, $\mathrm{CD} 4^{+} \mathrm{T}$ cells can either drive or constrain immune responses against tumors through the reciprocal function of effector and regulatory cell lineages, respectively $(2,3,20)$. Within tumors, Tregs can powerfully inhibit the accumulation and function of $\mathrm{CD} 4^{+}$and $\mathrm{CD} 8^{+}$effector $\mathrm{T}$ cells (3). We have previously found that BACH2 promotes Foxp $3^{+}$Treg development through repression of helper cell transcriptional programs $(8,21)$. We wished to determine whether BACH2-mediated tumor immuno- suppression was dependent upon its role in Treg development. In mice, Tregs can be identified by their expression of the TF Foxp3 (22-24). We observed substantially diminished Foxp3 ${ }^{+}$Treg populations in tumors from Bach2-deficient animals when expressed as either their frequency relative to total $\mathrm{CD} 4^{+} \mathrm{T}$ cells or $\mathrm{CD} 8^{+} \mathrm{T}$ cells within tumors or as their absolute number (Figure 3, A and B). The frequency of Foxp $3^{+}$Tregs was similarly reduced in both tumors and spleens of BACH2-deficient mice (Supplemental Figure 2), suggesting that defective Treg-mediated tumor immunosuppression in BACH2-deficient mice may correspond to the generalized Treg deficiency in these animals.

We wished to determine the relative contribution of Foxp $3^{+}$ Tregs to BACH2-mediated tumor immunosuppression. Since Tregs suppress immune function in an immunodominant fashion, phenotypes resulting from their deficiency are amenable to rescue by provision of WT Tregs. Tregs from mice expressing 
the human diphtheria toxin (DTx) receptor (DTR) under the control of the endogenous Foxp3 locus (Foxp $3^{\text {DTR }}$ mice) are sensitive to depletion following administration of DTx (25). To test whether BACH2-mediated immunosuppression is dependent upon the function of BACH2 in Treg development, we reconstituted irradiated $\mathrm{Rag}^{-/-}$mice with 1:1 mixtures of bone marrow (BM) cells from Foxp $3^{\text {DTR }}$ mice and either WT or Bach2-deficient mice, resulting in WT:Foxp $3^{\text {DTR }}$ and KO:Foxp $3^{\text {DTR }}$ mixed chimeric animals. This system allows experimental interrogation of Treg-dependent phenotypes attributable to Bach 2 deficiency, since Bach2-sufficient Tregs are provided by the Foxp $3^{\mathrm{DTR}} \mathrm{com}$ partment, except in cases in which DTx is administered. Using this system, we observed equivalent chimerism of WT and Bach2-deficient $\mathrm{CD} 4^{+}$and $\mathrm{CD} 8^{+} \mathrm{T}$ cells within reconstituted WT:Foxp $3^{\text {DTR }}$ and KO:Foxp $3^{\text {DTR }}$ mixed chimeric animals, respectively (Supplemental Figure 3, A and B), and near-complete DTx -induced depletion of Foxp3 $3^{+}$Tregs within Foxp $3^{\text {DTR }}$ compartments (Supplemental Figure 3C). Strikingly, BACH2-mediated tumor immunosuppression was dependent upon Foxp3+ Tregs, since impaired tumor growth and loss of immunosuppression was only observed when DTx was administered to KO:Foxp $3^{\text {DTR }}$ mice (Figure 3C). Growth of subcutaneous tumors in reconstituted Rag1 $^{-/}$radiation chimeras occurred with distinct kinetics compared with that in WT nonirradiated animals, as is consistent with our previous experience (our unpublished observations), and the duration of measurement in these experiments was limited by systemic effects of Treg depletion in mice reconstituted with mixtures of $\mathrm{KO}$ and Foxp $3^{\text {DTR }} \mathrm{BM}$ and administered DTx. Collectively, these findings suggest that BACH2-mediated tumor immunosuppression is dependent upon Foxp $3^{+}$Tregs.

We observed increased frequencies of $\mathrm{CD}^{+} \mathrm{T}$ cells and elevated production of IFN- $\gamma$ within tumors of Bach2-deficient animals. This led us to ask whether Treg-dependent tumor immunosuppression mediated by BACH2 is caused by suppression of $\mathrm{CD}^{+}$ $\mathrm{T}$ cells or IFN- $\gamma$. Strikingly, augmented tumor immunity observed upon administration of DTx to KO:Foxp $3^{\text {DTR }}$ mice was partially reversed upon depletion of $\mathrm{CD} 8^{+} \mathrm{T}$ cells and fully reversed upon simultaneous depletion of $\mathrm{CD}^{+} \mathrm{T}$ cells and blockade of IFN- $\gamma$ (Figure 3D). Thus, we conclude that $\mathrm{BACH} 2$ causes tumor growth through Treg-mediated suppression of $\mathrm{CD} 8^{+} \mathrm{T}$ cells and concomitant IFN- $\gamma$-dependent effector mechanisms.

In this study, we have found that the TF $\mathrm{BACH} 2$ is required for establishment of immunosuppression within tumors. Despite its activity in a diversity of innate and adaptive immune cell types, it predominantly exerts this function through its role in $\mathrm{CD} 4^{+}$Tregs, thus potently suppressing the antitumor activity of $\mathrm{CD} 8^{+} \mathrm{T}$ cells. These findings identify a molecular axis of tumor immunosuppression and provide targets for design of immune-based therapies aimed at reversing deleterious immunosuppression in cancer.

\section{Methods}

Additional details can be found in the Supplemental Methods.

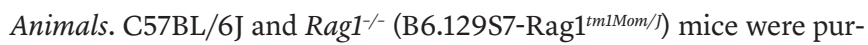
chased from The Jackson Laboratory. Bach2-deficient mice, which have been previously described (6), were backcrossed $>16$ times with C57BL/6 mice.

Data deposition. Data from whole-transcriptome analyses are deposited under GEO accession number GSE74653.

Statistics. Two-tailed Student's $t$ tests were used to calculate statistical significance of the difference in sample means. $P$ values of less than 0.05 were considered significant and are provided.

Study approval. Experiments were approved by the Institutional Animal Care and Use Committee of the National Cancer Institute and performed in accordance with NIH guidelines.

\section{Author contributions}

RR, RLE, DC, CAK, LG, and NPR conceived the study and wrote the manuscript. RR, RLE, DC, GM, ZY, HL, JHP, and PJ performed experiments. PJ and RR performed bioinformatic analyses. MS, FMG, ZY, YJ, DCP, AC, JGC, SJP, DS, EW, FMM, KO, and NPR edited the manuscript.

\section{Acknowledgments}

This research was supported by the Intramural Research Program of the US National Cancer Institute (ZIA BC010763) and the UK Biotechnology and Biological Sciences Research Council (grant BB/N007794/1). R. Roychoudhuri is supported by a Sir Henry Dale Fellowship, jointly funded by the Wellcome Trust and the Royal Society (grant 105663/Z/14/Z). The research was also supported by the Tiens Charitable Foundation, the NIH Center for Regenerative Medicine, and the Milstein Family Foundation.

Address correspondence to: Rahul Roychoudhuri, The Babraham Institute, Babraham Research Campus, Cambridge, CB22 3AT, UK. Phone: 44.1223.496315; E-mail: rahul.roychoudhuri@ babraham.ac.uk. Or to: Nicholas P. Restifo, National Cancer Institute, Rm. 3W-5762, Bld. 10/CRC, Bethesda, Maryland 20892, USA. Phone: 301.496.4904; E-mail: restifo@nih.gov.
1. Gabrilovich DI, Ostrand-Rosenberg S, Bronte V. Coordinated regulation of myeloid cells by tumours. Nat Rev Immunol. 2012;12(4):253-268.

2. Zou W. Regulatory T cells, tumour immunity and immunotherapy. Nat Rev Immunol. 2006;6(4):295-307.

3. Quezada SA, Peggs KS, Simpson TR, Allison JP. Shifting the equilibrium in cancer immunoediting: from tumor tolerance to eradication. Immunol Rev. 2011;241(1):104-118.

4. Rabinovich GA, Gabrilovich D, Sotomayor EM. Immunosuppressive strategies that are mediated by tumor cells. Annu Rev Immunol. 2007;25:267-296.
5. Gajewski TF, Schreiber H, Fu YX. Innate and adaptive immune cells in the tumor microenvironment. Nat Immunol. 2013;14(10):1014-1022.

6. Muto A, et al. The transcriptional programme of antibody class switching involves the repressor Bach2. Nature. 2004;429(6991):566-571.

7. Muto A, et al. Bach2 represses plasma cell gene regulatory network in B cells to promote antibody class switch. EMBO J. 2010;29(23):4048-4061.

8. Roychoudhuri R, et al. BACH2 represses effector programs to stabilize $\mathrm{T}(\mathrm{reg})$-mediated immune homeostasis. Nature. 2013;498(7455):506-510.

9. Kim EH, Gasper DJ, Lee SH, Plisch EH, Svaren J,
Suresh M. Bach2 regulates homeostasis of Foxp3 regulatory $\mathrm{T}$ cells and protects against fatal lung disease in mice. JImmunol. 2014;192(3):985-995.

10. Nakamura A, et al. Transcription repressor Bach2 is required for pulmonary surfactant homeostasis and alveolar macrophage function. JExp Med. 2013;210(11):2191-2204.

11. Sawcer S, et al. Genetic risk and a primary role for cell-mediated immune mechanisms in multiple sclerosis. Nature. 2011;476(7359):214-219.

12. Franke A, et al. Genome-wide meta-analysis increases to 71 the number of confirmed Crohn's disease susceptibility loci. Nat Genet. 
2010;42(12):1118-1125.

13. Cooper JD, et al. Meta-analysis of genomewide association study data identifies additional type 1 diabetes risk loci. Nat Genet. 2008;40(12):1399-1401.

14. Ferreira MA, et al. Identification of IL6R and chromosome $11 \mathrm{q} 13.5$ as risk loci for asthma. Lancet. 2011;378(9795):1006-1014.

15. Subramanian A, et al. Gene set enrichment analysis: a knowledge-based approach for interpreting genome-wide expression profiles. Proc Natl Acad Sci U S A. 2005;102(43):15545-15550.

16. Zhang N, Bevan MJ. CD8(+) T cells: foot soldiers of the immune system. Immunity. 2011;35(2):161-168.

17. Szabo SJ, Kim ST, Costa GL, Zhang X, Fathman
CG, Glimcher LH. A novel transcription factor, T-bet, directs Th1 lineage commitment. Cell. 2000;100(6):655-669.

18. Rutishauser RL, et al. Transcriptional repressor Blimp-1 promotes CD8(+) T cell terminal differentiation and represses the acquisition of central memory $\mathrm{T}$ cell properties. Immunity. 2009;31(2):296-308.

19. Mombaerts P, Iacomini J, Johnson RS, Herrup K, Tonegawa S, Papaioannou VE. RAG-1-deficient mice have no mature B and T lymphocytes. Cell. 1992;68(5):869-877.

20. Roychoudhuri R, Eil RL, Restifo NP. The interplay of effector and regulatory $\mathrm{T}$ cells in cancer. Curr Opin Immunol. 2015;33:101-111.

21. Vahedi G, et al. Super-enhancers delineate disease-associated regulatory nodes in T cells. Nature. 2015;520(7548):558-562.

22. Hori S, Nomura T, Sakaguchi S. Control of regulatory $\mathrm{T}$ cell development by the transcription factor Foxp3. Science. 2003;299(5609):1057-1061.

23. Khattri R, Cox T, Yasayko SA, Ramsdell F. An essential role for Scurfin in $\mathrm{CD} 4{ }^{+} \mathrm{CD} 25^{+} \mathrm{T}$ regulatory cells. Nat Immunol. 2003;4(4):337-342.

24. Fontenot JD, Gavin MA, Rudensky AY. Foxp3 programs the development and function of $\mathrm{CD}^{+} \mathrm{CD} 25^{+}$regulatory T cells. Nat Immunol. 2003;4(4):330-336.

25. Kim JM, Rasmussen JP, Rudensky AY. Regulatory T cells prevent catastrophic autoimmunity throughout the lifespan of mice. Nat Immunol. 2007;8(2):191-197. 